\title{
Workplace health promotion using a digital health programme with monthly telecoaching to improve clinical and lifestyle-related outcomes
}

\author{
Authors: Austen El-Osta, ${ }^{\mathrm{A}}$ Emmanouil Bagkeris, ${ }^{\mathrm{B}}$ Dalton Coker, ${ }^{\mathrm{A}}$ Monal Wadhera, ${ }^{\mathrm{C}}$ Nicola Hursthouse, ${ }^{\mathrm{D}}$ \\ Alexander Heaton ${ }^{D}$ and Azeem Majeed ${ }^{A}$
}

\begin{abstract}
Aims
To evaluate the effectiveness of a workplace digital health promotion programme with monthly telecoaching on various lifestyle and clinical outcome measures.
\end{abstract}

\section{Methods}

We recruited 103 participants from an occupational setting in the UK. Participants were assessed at baseline, and 3 and 6 months following intervention to determine the effect of an employer sponsored digital telecoaching intervention on various lifestyle and clinical outcome measures.

\section{Results}

A pragmatic sample size of 103 participants from a wide demographic background was recruited at baseline, with 95 participants ( $92 \%$ ) completing the programme by 6 months. Almost all participants showed a significant improvement in the health score and 24 secondary outcome measures including $\mathrm{HbA1c}$, blood pressure and reduction in smoking. Linear regression model showed that after adjusting for age and gender, the LiveSmart health score appeared to significantly improve by 6.22 units at 3 months ( $95 \%$ confidence interval (CI) 4.38 to 8.06) and by 7.73 units at 6 months ( $95 \%$ CI 5.75 to 9.71), compared with baseline visit, $p<0.001$.

\section{Conclusion}

The LiveSmart digital health promotion programme with monthly coaching was a largely successful intervention programme achieving good traction with participants and significant improvement in a number of key outcomes, including lower $\mathrm{HbA} 1 \mathrm{c}$ and reduced salt intake among other clinical and healthy lifestylerelated behaviours. A simplistic approach to modelling suggests that the programme has a good return on investment overall and offers a number of tangible and intangible benefits to the service user and the employer, and in the context of the wider health economy. A larger study with a longer follow-up is recommended to better understand the cost-effectiveness of health promotion interventions in the occupational setting.

\section{Conflict of interest statement}

None.

Authors: ${ }^{\mathrm{A}}$ Imperial College; ${ }^{\mathrm{B}} \mathrm{UCL} ;{ }^{\mathrm{C}}$ Chief Medical Officer;

DLiveSmart, London, UK 